\title{
Geometrical Effects on the Magnetic Properties of Nanoparticles
}

\author{
Cono Di Paola, ${ }^{\dagger,+}$ Roberto D’Agosta, ${ }^{*}, \S^{\prime},, \|$ and Francesca Baletto ${ }^{*}, \dagger$ \\ ${ }^{\dagger}$ Department of Physics, King's College London, WC2R 2LS, London, United Kingdom \\ ${ }^{*}$ Department of Earth Sciences, University College London, WC1E 6BT, London, United Kingdom \\ ${ }^{\S}$ Nano-bio Spectroscopy Group and ETSF, Universidad del País Vasco, CFM CSIC-UPV/EHU, E-20018 San Sebastián, Spain \\ "Ikerbasque, Basque Foundation for Science, E-48013, Bilbao, Spain
}

\section{Supporting Information}

ABSTRACT: Elucidating the connection between shape and properties is a challenging but essential task for a rational design of nanoparticles at the atomic level. As a paradigmatic example we investigate how geometry can influence the magnetic properties of nanoparticles, focusing in particular on platinum clusters of $1-2 \mathrm{~nm}$ in size. Through first-principle calculations, we have found that the total magnetization depends strongly on the local atomic arrangements. This is due to a contraction of the nearest neighbor distance together with an elongation of the second nearest neighbor distance, resulting in an interatomic partial charge transfer from the atoms lying on the subsurface layer (donors) toward the vertexes (acceptors).

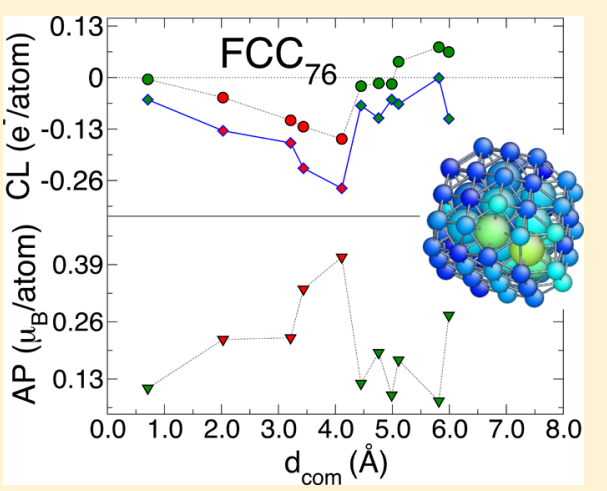

KEYWORDS: Platinum, nanoclusters, magnetism, deformation, geometry

$\mathrm{T}$ he great scientific and technological advances in assembling better electrical, catalytic, magnetic, and optical devices have stimulated intensive research at the nanoscale, where small and finite size metallic nanoparticles (mNP) offer the natural building blocks for a bottom-upapproach. $^{1-5}$ The process of designing and tailoring $\mathrm{mNP}$ benefits from the knowledge of how certain physical properties or chemical activities depend on the size and shape of the nanoparticle itself. This is a highly nontrivial endeavor due to the strong dependence of the electronic structure on the interatomic distances, which are highly distorted especially in noncrystallographic geometries. ${ }^{6,7}$ For example, it has recently been shown that some catalytic activity can be rationalized in terms of a generalized coordination number which depends on the local atomic geometrical order up to the second nearest neighbor (NN) distance. ${ }^{8}$ Similarly, it would be of great technological interest to provide a geometrical interpretation of the appearance and enhancement of magnetism in mNP. Indeed, the challenge in the magnetic component industry is to design and assemble metallic nanostructures with controlled geometry maximizing the packing density and manipulating their mutual magnetic interaction, e.g., through doping with natural magnets as $\mathrm{Fe}$ and Co. ${ }^{9}$ Looking at the magnetic properties of transition metal nanosystems, it has been realized that partially occupied and energetically degenerate d-orbitals retain to some extent their atomic character. ${ }^{10}$ This can lead to high magnetic moments even in nonmagnetic bulk species, such as $\mathrm{Au}, \mathrm{Pd}$, and $\mathrm{Pt}$ due to a nonuniform spin distribution in the clusters. ${ }^{10-28}$ Some of these results are interpreted starting from a theory of "super-atom" where the cluster shows some of the physical properties of its components. ${ }^{10}$ However, this model can be successfully applied only to relatively small clusters, below the nanometer diameter, and does not explain the behavior observed in larger clusters where there is still only a single magnetic domain. At the same time, the standard approach to ferro-magnetism based on the coexistence of different magnetic domains and their mutual alignment can only be applied to particles larger than $100 \mathrm{~nm} .^{29}$

As platinum offers a quite unique feature, namely, it is nonmagnetic in bulk and magnetic at the nanoscale, we have focused our attention on $\mathrm{Pt}$ clusters in a wide size diameter range between 1 and $2 \mathrm{~nm}$. The appearance of a magnetic order in Pt nanoparticles has been addressed in recent experiments. In superconducting quantum interference device experiments, the magnetic properties of $\mathrm{Pt}$ nanoparticles synthesized by wetchemistry techniques have been observed to depend strongly on the morphology where branched nanosystems are ferromagnetic, whereas spherical symmetry induces paramagnetism. $^{25,30}$ Breaking symmetry and inducing charge transfer enhance the total magnetic moment: This is the case of surfactants in comparison with amino-coated $\mathrm{Pt}$ nanomaterials, where the charge transfer is less efficient. ${ }^{17,23}$ Deliberately introducing certain geometrical deformations in Pt nanowires should be considered as a further fundamental approach devoted to manipulate and tune the magnetic

Received: March 2, 2016

Revised: $\quad$ March 22, 2016

Published: March 23, 2016 
behavior in Pt nanosystems. ${ }^{31,32}$ Finally, recent experiments in atomic Pt contacts show pronounced magnetic conductance depending on their atomic configurations. ${ }^{33}$

From a theoretical point of view, extensive numerical simulations based on density functional theory (DFT) have been performed to understand the magnetic nature of $\mathrm{Pt}$ thin films ${ }^{34,35}$ and nanowires, where the appearance of ferromagnetism has been predicted only for certain structures. ${ }^{36,37}$ Nonetheless, only a few studies have been carried out on free $\mathrm{Pt}$ nanoparticles. In particular, magnetism in Pt nanoclusters has only been associated with partially filled d-states. ${ }^{38}$ At the same time, the geometrical distortions characteristic of a finite nanoobject can lead to a shift between minority and majority spin bands, and thus less coordinated sites may show a higher magnetic moment. ${ }^{39,40}$ Indeed, what is the contribution of surface, vertex, and core atoms and how an enhancement of the magnetism in Pt clusters is associated with geometrical factors have never been addressed from an atomistic point of view.

Using Pt clusters as our playground, we here show that the finite magnetic moment of $\mathrm{mNP}$ is related to the geometrical features of the nanoparticle itself. We observe that each cluster does present ferro-magnetism and that highly symmetric icosahedral configurations usually return the largest magnetic moment, especially for the larger sizes under consideration here. We associate this observation to (a) a minority spin charge moves from the subvertexes to their vertexes due to a strong contraction of their bond length and (b) the ability of the vertexes to retain these extra spins in the majority spin channel due to a slightly elongated second NN distance within the surface shell with respect to their bulk values. Finally, we are able to relate the differences among morphologies to the second nearest neighbor distances around each vertex, with a contraction only toward atoms on a (100). Magnetic depletion (e.g., $\mathrm{Pt}_{147}$ crystallographic cuboctahedra) is possible and it is related to a different contraction/elongation of the first and second NN bond lengths to deviations from the bulk value that are propagating up to the second $\mathrm{NN}$ distances.

The magnetic behavior of $\mathrm{Pt}_{N}$, where $N$ is the total number of atoms in the cluster, is calculated using a plane-wave code based on density functional theory included in the Quantum ESPRESSO package ${ }^{41}$ where all the configurations are ionically relaxed using the Broyden-Fletcher-Goldfarb-Shann's procedure and the total magnetic moments are obtained performing unrestricted local spin-polarized simulations. (See Supporting Information for details about the parameters used in the calculations.) Furthermore, our analysis is not restricted to the magic sizes of icosahedral ( $\mathrm{Ih}$ ), decahedral (Dh), and cuboctahedra (CO) shapes, corresponding to $13,55,147$, and 309 atoms of the above polyhedra, but it includes: uncompleted and polyicosahedral shapes at 38 and 76 atoms, since they have been found to be promising structural motifs for $\mathrm{CoPt}$ systems; $^{42}$ the Marks twinning of decahedra at 39 and 75 atoms, and a FCC motif at 76 atoms which corresponds to an incomplete octahedron at 85 atoms with a stacking fault and cuts to obtain (100) facets. (See Supporting Information for examples of all the structures considered here.)

The calculated macroscopic magnetism of the considered $\mathrm{Pt}$ morphologies is shown in Figure 1, as the atomic averaged total magnetization (aTM) plotted versus size. For the sake of comparison, the dimer and the bulk values are added as reference points. A ferromagnetic ordering is always observed in each nanomorphology, as shown in Figure 1 for the CO, Ih, and $\mathrm{Dh}$ arrangements at 55 atoms. In the Supporting

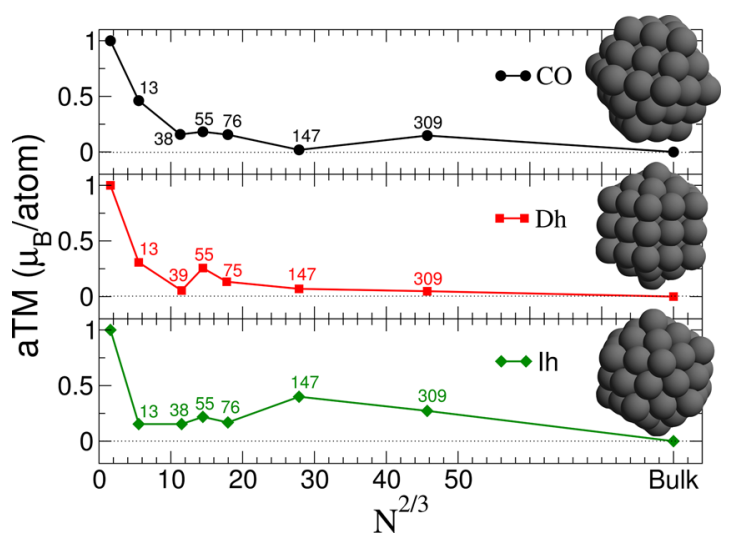

Figure 1. Atomic averaged magnetization, aTM, in $\mu_{\mathrm{B}} /$ atom as a function of size $N^{2 / 3}$. The dimer and bulk values are included for comparison. Each point is also labeled according to the total number of atoms in the cluster. Top panel CO and FCC-like structures; middle panel Dh; bottom Ih. The aTM is calculated as the total magnetization, $M_{\text {tot, }}$ divided by the total number of atoms, $N$, i.e., aTM $=M_{\text {tot }} / N=\int n^{\uparrow}(\mathbf{r})-n^{\downarrow}(\mathbf{r}) d \mathbf{r} / N$, where the integral runs over all space, while $n^{\uparrow}(\mathbf{r})$ and $n^{\downarrow}(\mathbf{r})$ are the electron charge densities for spin up and spin down, respectively. For each of the investigated clusters we have provided an example of the structure with 55 atoms.

Information, we have included a figure with the cross section of a $\mathrm{Ih}_{147}$ to help visualizing its shell structure. For the $\mathrm{CO}$ system, top panel of Figure 1, the averaged total magnetic moment presents a flat region at $0.15-0.20 \mu_{\mathrm{B}}$ /atom between 38 and 309 atoms, with an unexpected dip at 147 atoms. Decahedra peak in their aTM at 55 atoms with $0.25 \mu_{\mathrm{B}}$ /atom and then slowly decay to the asymptotic bulk para-magnetism. On the other hand, for Ih patterns the averaged total magnetic moment tops around 150 atoms after an almost constant behavior at smaller sizes. To test the generality of our analysis, we have examined different initial starting magnetizations. For simplicity, most of the calculations started with a ferromagnetic initial states. At the same time, an antiferromagnetic ordering, where the core atoms had magnetization opposite with respect to the shell and subshell atoms has been used as initial configuration. After full electron relaxation, the ground state has been found to be ferromagnetic again. For a more systematic analysis of spin restricted calculations for $\mathrm{Pt}_{13}$ with different structural motifs has been reported elsewhere. ${ }^{43}$ We have also performed noncollinear calculations for clusters at 13 and 55 atoms. We have observed that once fully relaxed, a ferromagnetic order emerges, and the spins are essentially aligned. Although running a full noncollinear calculation at larger sizes is for us computational unfeasible, we do not expect the scenario to change significantly.

The appearance of the magnetic behavior in $\mathrm{mNP}$ can be rationalized in terms of two contributions: an intra- and an interatomic charge transfer (Heisenberg exchange). The intraatomic charge transfer is induced by an intraband splitting around the Fermi energy between majority and minority populations. The interatomic contribution stems from the charge transfer between adjacent atoms from the minority band of the donor to the majority band of the acceptor. At the same time, minority electrons can transfer from the acceptor to the majority band of the donors, therefore increasing the overall atomic polarization. Mohn suggested that a further majority/ minority band splitting can be originated by a strong distortion of the second nearest neighbor distances with respect to their 

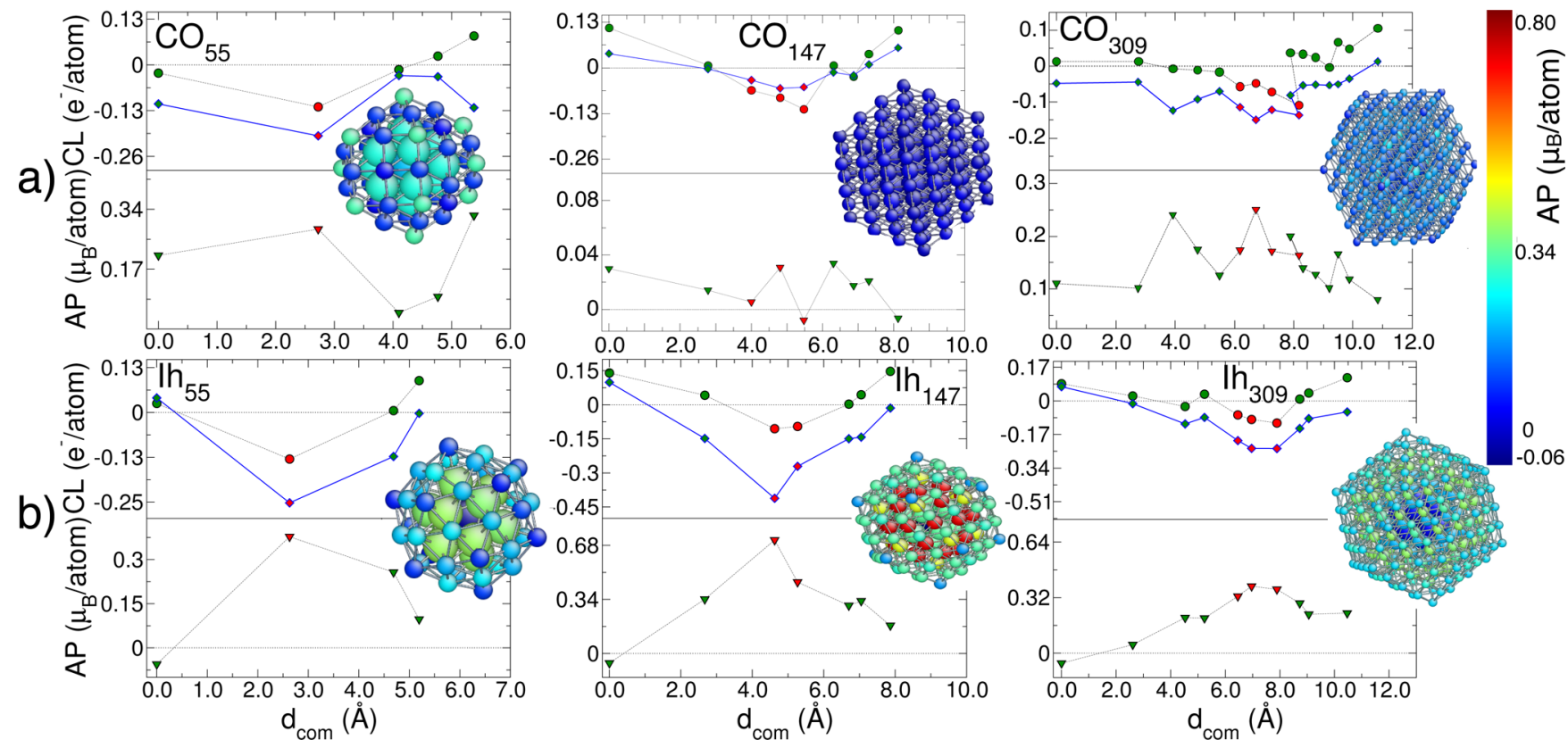

Figure 2. AP (triangle), total $\mathrm{CL}^{\mathrm{T}}$ (circle), and minority $\mathrm{CL}^{\downarrow}$ (diamond) charge losses plotted against the radial distance, $d_{\text {com }}$ for (a) CO and (b) Ih shapes at various sizes up to 309 atoms. The symbol color indicates: red $=$ subsurface, green $=$ other atoms. In the snapshots, atoms are colored according to their AP in temperature gradient as shown at the right. The quantities showed here have been derived in the following way: $\mathrm{CL}^{\downarrow}=a_{\mathrm{B}}^{\downarrow}-$ $e_{\mathrm{S}}^{\downarrow}\left(\mathrm{CL}^{\uparrow}=a_{\mathrm{B}}^{\uparrow}-e_{\mathrm{S}}^{\uparrow}\right), \mathrm{CL}^{\mathrm{T}}=a_{\mathrm{B}}-e_{\mathrm{S}} \equiv \mathrm{CL}^{\uparrow}+\mathrm{CL}^{\downarrow}$, and $\mathrm{AP}=\mathrm{CL}^{\uparrow}-\mathrm{CL}^{\downarrow}$, where $e_{\mathrm{S}}=10$ and $e_{\mathrm{S}}^{\downarrow}=e_{\mathrm{S}}^{\uparrow}=5$ are the total and partial valence electrons in the nonmagnetic bulk, $a_{\mathrm{B}}$ and $a_{\mathrm{B}}^{\downarrow}\left(a_{\mathrm{B}}^{\uparrow}\right)$ are the total and partial atomic charges calculated via the Bader analysis, respectively.

bulk value. ${ }^{40}$ Here, we demonstrate that the ferromagnetic behavior, high aTM values, in Pt nanoparticles arises from the interatomic charge transfer between vertexes lying on consecutive shells, and therefore is strongly influenced by the local geometry. The finite and high atomic polarization (AP), averaged over atoms at equivalent sites, is not uniquely associated with atoms with a low coordination number (surface atoms), but instead it peaks at the subsurface, and only in a couple of case surface atoms show a similar AP.

To quantify the relationship between the local environment and magnetic properties, atomic charges are computed performing a Bader analysis ${ }^{44,45}$ over the total as well as the majority and minority spin charge densities. In this way, we have been able to estimate the intra- and interatomic charge transfers in terms of the total atomic charge loss $\left(\mathrm{CL}^{\mathrm{T}}\right)$ and $\mathrm{AP}$ obtained as the difference between the majority and minority spin charge losses, $\left(\mathrm{CL}^{\uparrow}, \mathrm{CL}^{\downarrow}\right), \mathrm{AP}=\mathrm{CL}^{\uparrow}-\mathrm{CL}^{\downarrow}$. The total and minority charge losses as well as AP are reported in Figure 2 as a function of the radial distance from the center of mass of the clusters $d_{\text {com }}$ for $\mathrm{CO}$, and Ih, respectively. The same quantities for the $\mathrm{Dh}$ cluster family are reported in the Supporting Information. A finite AP due to an intra-atomic charge transfer would imply a vanishing $\mathrm{CL}^{\mathrm{T}}=0$ because no charge is transferred between atoms. The observation of a finite $\mathrm{CL}^{\mathrm{T}}$ implies that the interatomic charge transfer contribution is the most effective in Pt nanoparticles. Indeed, we show how the subvertexes play an important role in the distribution of the local magnetism and that there is a net charge transfer from the subshell to the outermost shell as depicted in Figure 3. We would like to comment that the total charge transferred per atom per shell, in the external shell is about constant, around 0.03-0.04 electron per shell per atom. Obviously, the amount of electrons per atom transferred from the subshell decreases with increasing the size of the cluster.

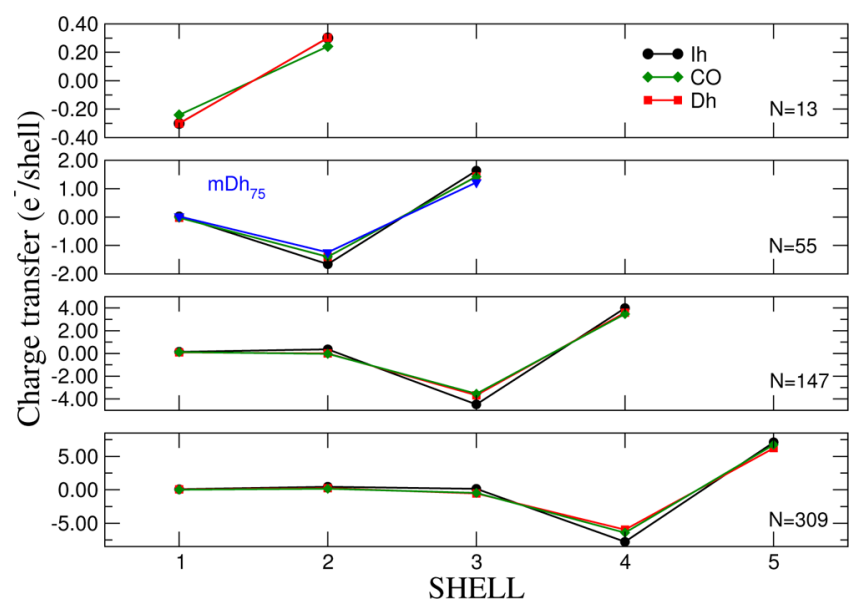

Figure 3. Total charge transfer (in electron per shell) between neighboring shells. A similar behavior is observed in all of the nanoparticles considered.

Let us give a closer look at how the local environment influences the interatomic charge transfer. From the smallest sizes, but where three shells could be drawn as shown in Figure 3 , the subvertexes are found to act as donors (negative $\mathrm{CL}^{\mathrm{T}}$, see red dots in Figure 2) of partial charge and this charge exchange is generally observed to be carried out by the minority population. On the other hand, vertexes tend to act as acceptors (positive $\mathrm{CL}^{\mathrm{T}}$ ) with the majority band accommodating mostly of the received charge ( $\mathrm{CL}^{\downarrow}$ is negligible). The interatomic charge transfer toward the innermost shells seems to be more complicated but is still substantially dominated by the subsurface atomic behavior. Ih clusters, independently of their size, show a quite clear behavior both in the AP and CLs; see Figure $2 \mathrm{~b}$. The subshell is highly polarized with a net charge transfer toward the vertexes of the cluster, leading to a 
significant magnetization. The $\mathrm{CO}$ shape instead shows a more varied behavior; see Figure 2a. For $\mathrm{CO}_{55}$ and $\mathrm{CO}_{309}$ there is a peak of $\mathrm{AP}$ in the subshell and some remnant $\mathrm{AP}$ at the vertexes; also charge is transferred from the core and subshells to the surface. At both sizes there is a similar magnetization per atom. For $\mathrm{CO}_{147}$, on the other hand, the $\mathrm{AP}$ is almost vanishing for each shell, and more significantly the magnetization essentially disappears. We should add that, in all our calculations, we observe a discrepancy as tiny as a small fraction of a Bohr magneton per atom between the total and absolute magnetization, the latter being defined as $\int \ln \uparrow(\mathbf{r})-$ $n^{\downarrow}(\mathbf{r}) \mid \mathrm{dr}$. We attribute these differences to negligible numerical fluctuations in the spin densities. Hence, the changes of the total magnetization among morphologies and sizes cannot be associated with the appearance of regions with opposite magnetization.

To understand how the magnetization depends on the local geometry, we have investigated the distance distribution of first and second nearest neighbors of each vertex and subvertex, as illustrated in Figure 4. In panel b of Figure 4, for $\mathrm{Pt}_{147}$ and $\mathrm{Pt}_{309}$ and all of the considered geometrical motifs, we tabulate the

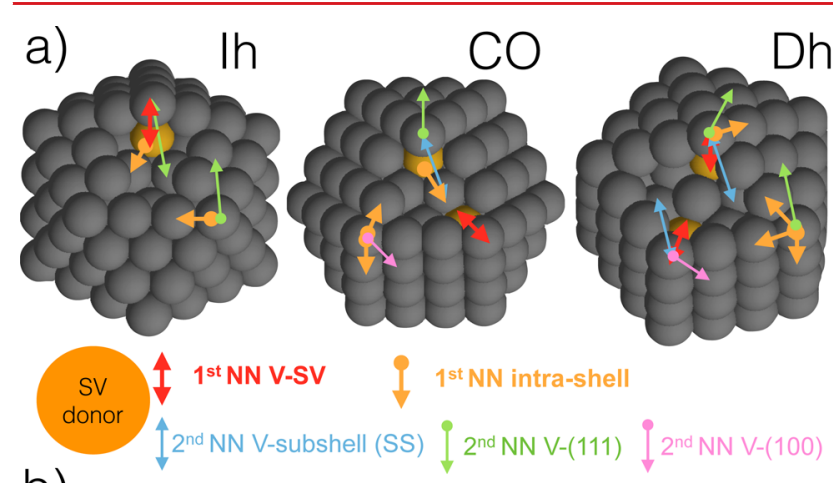

b)

\begin{tabular}{|c|c|c|c|c|c|c|c|}
\hline & & $\mathrm{Ih}_{147}$ & Ih 309 & $\mathrm{CO}_{147}$ & $\mathrm{CO}_{309}$ & $\mathrm{Dh}_{147}$ & Dh309 \\
\hline \multirow{4}{*}{$\begin{array}{l}1^{\text {st }} \\
\mathrm{NN}\end{array}$} & V-SV & $8 \%$ & $7.9 \%$ & $4.7 \%$ & $5.9 \%$ & $(6.5: 8.7) \%$ & $(1.8: 7.4) \%$ \\
\hline & SV-core & $8 \%$ & $5.6 \%$ & $6 \%$ & $6.7 \%$ & $(6.4: 7.4) \%$ & $(3.6: 5.2) \%$ \\
\hline & $\begin{array}{l}\text { V intra- } \\
\text { shell }\end{array}$ & $1.7 \%$ & $2.7 \%$ & $3.1 \%$ & $2.8 \%$ & $(2.4: 5.2) \%$ & $(1.1: 5) \%$ \\
\hline & $\begin{array}{l}\text { SV } \\
\text { intra- } \\
\text { shell }\end{array}$ & $1.9 \%$ & $2.8 \%$ & $4 \%$ & $3.6 \%$ & $(1.7: 7.6) \%$ & $(1.3: 3) \%$ \\
\hline \multirow{3}{*}{$\begin{array}{l}2^{\text {nd }} \\
\text { NN }\end{array}$} & V-SS & $-15 \%$ & $-16 \%$ & $-16 \%$ & $-16 \%$ & $-(11: 15) \%$ & $-(15: 19) \%$ \\
\hline & V-(111) & $-19 \%$ & $-19 \%$ & $-17 \%$ & $-18 \%$ & $-(15: 18) \%$ & $-(18: 19) \%$ \\
\hline & $V=(100)$ & NA & NA & $5 \%$ & $4.9 \%$ & $6.4 \%$ & $5 \%$ \\
\hline
\end{tabular}

Figure 4. (a) Schematic network of NN average distance of vertex (V) and subvertex (SV). Double arrows stand for atoms lying on different shells, while single arrow refers to atoms lying on the same shell. Schematic second NN network around V: toward (111) surface atoms, common for both Ih and CO; atoms belonging to (100) facets, only for CO. Also shown is a color code to easily identify the nature of the arrow. (b) Taking the DFT bulk lattice parameter as reference, the Table reports the relative distortion of the first $\mathrm{NN}$ distances: coreSV, SV-V, V-intrashell, SV-intrashell; and the second NN distances: $\mathrm{V}$-sub shell (SS), V-(111), and V-(100) for the considered shapes at 147 and 309 atoms. A positive (negative) value corresponds to a contraction (elongation). relative distortion with respect to the bulk lattice parameter of the average bond length between vertex and subvertex; subvertex and subsub-vertex (core); the average first $\mathrm{NN}$ distances around vertex and subvertex, respectively; the average distance between vertex and its second neighbors lying on the subshell, on the (111) and, if it is the case, on (100) facets, respectively. For the decahedral shapes, characterized by two nonequivalent vertexes, one lying on a 5-fold axes and the other shared by $(100) /(111)$ facets, we report the range of possible values. All of the geometries exhibit a contraction of the first NN distance with respect to the bulk value. We observe a stronger compression along the radial direction coresubvertex-vertex (labeled SV and V in Figure 4, respectively), than in the intrashell directions. A high aTM is observed in all the clusters having a large discrepancy between the radial and intrashell contractions of the first shell of neighbors. This justifies the retention by the vertexes of the extra charge likely in the majority spin population, which is at the origin of a finite magnetic moment. A further look at the $\mathrm{CO}_{147}$ reveals that the intrashell and the intershell separations are indeed equally shortened with respect to their bulk value. These similar distortions result in the inability of the vertexes to retain the extra charge, reducing the effective magnetism in $\mathrm{CO}_{147}$ as reported in Figure 2. Quantitatively, the vanishing aTM is justified by the fact that for $\mathrm{CO}_{147}, \mathrm{CL}^{\uparrow} \simeq \mathrm{CL}^{\downarrow}$ (as can be seen from Figure 2) and $\mathrm{AP} \simeq 0$. Moreover, the second $\mathrm{NN}$ distances between vertex and (111) facet atoms are always elongated, leading to a reduction of the intrashell charge transfer. This effect can further enhance the majority/minority band splitting. On the other hand, in $\mathrm{CO}$ and $\mathrm{Dh}$, it is partially compensated because the bond-length of vertexes with their second neighbors lying on (100) facets is contracted.

To summarize, spin-polarized density functional simulations are carried out to calculate the total magnetization of crystallographic and noncrystallographic Pt nanoparticles for sizes up to 309 atoms. It is observed that an enhanced magnetism appears especially for large (above 100 atoms) icosahedral Pt clusters, while in cuboctahedra and FCC-like the average magnetization has an almost flat behavior from 36 to 309 atoms (with the notable exception of 147) and in decahedral it peaks at small sizes and then rapidly decreases. Through a Bader estimate of the effective atomic charges, we have found that the atomic magnetic properties of $\mathrm{Pt}_{N}$, where $N$ is the number of the atoms in the cluster, are ruled mainly by an Heisenberg interatomic contribution as the total charge loss is not vanishing. This effect is related to a net charge transfer between subvertexes (donors) and vertexes (acceptors), and it is strongly influenced by the local geometrical environment associated, in particular, with the contraction of the intershell distance between the two uppermost layers and the elongation of the second nearest neighbor distances between vertexes and (111) atoms. The presence of (100) facets seems instead to reduce the ability of the vertexes to retain the extra charge. Although an extension across the periodic table is beyond the scope of this paper, we believe that the role of subvertexes as donors, vertexes as acceptors, and the appearance of an interatomic charge transfer from the sub- toward the outer-shell to be generic as due to contraction/elongation of first and second neighbor distances and the presence of (100) facets. We hope that this work will boost further experimental and theoretical investigations aimed at showing how the morphology might influence the physical properties at the nanoscale. 


\section{ASSOCIATED CONTENT}

\section{S Supporting Information}

The Supporting Information is available free of charge on the ACS Publications website at DOI: 10.1021/acs.nanolett.6b00916.

Details of the numerical parameters and techniques used; atomic polarization and charge losses for the $\mathrm{Dh} \mathrm{Pt}$ nanoparticles; examples of the structure of all the atomic clusters considered; a list of acronyms used (PDF)

\section{AUTHOR INFORMATION}

\section{Corresponding Authors}

*E-mail: roberto.dagosta@ehu.es.

*E-mail: francesca.baletto@kcl.ac.uk.

Notes

The authors declare no competing financial interest.

\section{ACKNOWLEDGMENTS}

C.D.P. and F.B. have been supported by the U.K. research council EPSRC, under Grant No. EP/GO03146/1. R.D'A. acknowledges support by DYN-XC-TRANS (Grant No. FIS2013-43130-P) and NANOTherm (CSD2010-00044) of the Spanish Ministerio de Economia y Competitividad, the Grupo Consolidado UPV/EHU del Gobierno Vasco (Grant No. IT578-13), and Grant No. MV-2015-1-17 of the Diputacion Foral de Guipuzkoa, S. Volz at the Ecole Centrale de Paris for his hospitality while this work was completed, and the kind hospitality of the Physics Department of King's College London. We thank A. Comisso for useful suggestions and stimulating discussions, D. Lasa for technical support, and acknowledge the computational time from DIPC.

\section{REFERENCES}

(1) Sun, S.; Murray, C.; Weller, D.; Folks, L.; Moser, A. Science 2000, 287, 1989-1992.

(2) Nishihata, Y.; Mizuki, J.; Akao, T.; Tanaka, H.; Uenishi, M.; Kimura, M.; Okamoto, T.; Hamada, N. Nature 2002, 418, 164.

(3) Koga, K.; Ikeshoji, T.; Sugawara, K. Phys. Rev. Lett. 2004, 92, 115507.

(4) Urban, J. J.; Talapin, D. V.; Shevchenko, E. V.; Kagan, C. R.; Murray, C. B. Nat. Mater. 2007, 6, 115.

(5) Shaw, C.; Fernig, D.; Levy, R. J. Mater. Chem. 2011, 21, 12181.

(6) Baletto, F.; Ferrando, R. Rev. Mod. Phys. 2005, 77, 371.

(7) Di Paola, C.; Baletto, F. Eur. Phys. J. D 2013, 67, 49.

(8) Calle-Vallejo, F.; Tymoczko, J.; Colic, V.; Vu, Q. H.; Pohl, M. D.; Morgenstern, K.; Loffreda, D.; Sautet, P.; Schuhmann, W.; Bandarenka, A. S. Science 2015, 350, 185-189.

(9) Frey, N.; Sun, S. Inorganic Nanoparticles: Synthesis, Applications, and Perspectives; CRC Press-Taylor and Francis Group, LLC, 2010.

(10) Medel, V.; Reveles, J.; Khanna, S.; Chauhan, V.; Sen, P.; Castleman, A. Proc. Natl. Acad. Sci. U. S. A. 2011, 108, 10062.

(11) Nealon, G. L.; Donnio, B.; Greget, R.; Kappler, J.-P.; Terazzi, E.; Gallani, J.-L. Nanoscale 2012, 4, 5244.

(12) Singh, R. J. Magn. Magn. Mater. 2013, 346, 58-73.

(13) Shinohara, T.; Sato, T.; Taniyama, T. Phys. Rev. Lett. 2003, 91, 197201.

(14) Sampedro, B.; Crespo, P.; Hernando, A.; Litran, R.; Lopez, J.; Cartes, C.; Fernandez, A.; Ramirez, J.; Calbet, J.; Vallet, M. Phys. Rev. Lett. 2003, 91, 237203.

(15) Yamamoto, Y.; Miura, T.; Nakae, Y.; Teranishi, T.; Miyake, M.; Hori, H. Phys. B 2003, 329, 1183.

(16) Garcia, M. A.; Ruiz-Gonzalez, M. L.; de la Fuente, G. F.; Crespo, P.; Gonzalez, J. M.; Llopis, J.; Gonzalez-Calbet, J. M.; Vallet-Regi, M.; Hernando, A. Chem. Mater. 2007, 19, 889.
(17) Zhang, H.-T.; Ding, J.; Chow, G.-M. Langmuir 2008, 24, 375.

(18) Crespo, P.; Litran, R.; Rojas, T.; Multigner, M.; de la Fuente, J.; Sanchez-Lopez, J.; Garcia, M.; Hernando, A.; Penades, S.; Fernandez, A. Phys. Rev. Lett. 2004, 93, 087204.

(19) Yamamoto, Y.; Miura, T.; Suzuki, M.; Kawamura, N.; Miyagawa, H.; Nakamura, T.; Kobayashi, K.; Teranishi, T.; Hori, H. Phys. Rev. Lett. 2004, 93, 116801.

(20) Cademartiri, L.; Kitaev, V. Nanoscale 2011, 3, 3435.

(21) Kowlgi, K. N. K.; Koper, G. J. M.; Picken, S. J.; Lafont, U.; Zhang, L.; Norder, B. Langmuir 2011, 27, 7783-7787.

(22) Bergeron, D. E.; Roach, P. J.; Castleman, A., Jr.; Jones, N.; Khanna, S. N. Science 2005, 307, 231.

(23) Enders, A.; Skomski, R.; Honolka, J. J. Phys.: Condens. Matter 2010, 22, 433001.

(24) Kumar, V.; Kawazoe, Y. Phys. Rev. B: Condens. Matter Mater. Phys. 2008, 77, 205418.

(25) Salazar-Alvarez, G.; Qin, J.; Šepelák, V.; Bergmann, I.; Vasilakaki, M.; Trohidou, K. N.; Ardisson, J. D.; Macedo, W. A. A.; Mikhaylova, M.; Muhammed, M.; Baró, M. D.; Nogués, J. J. Am. Chem. Soc. 2008, 130,13234

(26) Singh, R.; Kroll, P. Phys. Rev. B: Condens. Matter Mater. Phys. 2008, 78, 245404.

(27) Langenberg, A.; Hirsch, K.; Lawicki, A.; Zamudio-Bayer, V.; Niemeyer, M.; Chmiela, P.; Langbehn, B.; Terasaki, A.; Issendorff, B. V.; Lau, J. T. Phys. Rev. B: Condens. Matter Mater. Phys. 2014, 90, 184420.

(28) Sebetci, A. Phys. Chem. Chem. Phys. 2009, 11, 921-925.

(29) Issa, B.; Obaidat, I. M.; Albiss, B. A.; Haik, Y. Int. J. Mol. Sci. 2013, 14, 21266.

(30) Liu, X.; Bauer, M.; Bertagnolli, H.; Roduner, E.; van Slageren, J.; Phillipp, F. Phys. Rev. Lett. 2006, 97, 253401.

(31) Teng, X.; Han, W.-Q.; Ku, W.; Hucker, M. Angew. Chem., Int. Ed. 2008, 47, 2055-2058.

(32) Chen, A.; Holt-Hindle, P. Chem. Rev. 2010, 110, 3767.

(33) Strigl, F.; Espy, C.; Bückle, M.; Scheer, E.; Pietsch, T. Nat. Commun. 2015, 6, 6172.

(34) Blugel, S. Phys. Rev. B: Condens. Matter Mater. Phys. 1995, 51, 2025.

(35) Niklasson, A.; Mirbt, S.; Skriver, H.; Johansson, B. Phys. Rev. B: Condens. Matter Mater. Phys. 1997, 56, 3276.

(36) Smogunov, A.; Dal Corso, A.; Delin, A.; Weht, R.; Tosatti, E. Nat. Nanotechnol. 2008, 3, 22.

(37) Smogunov, A.; Dal Corso, A.; Tosatti, E. Phys. Rev. B: Condens. Matter Mater. Phys. 2008, 78, 014423.

(38) Luo, W.; Pennycook, S. J.; Pantelides, S. T. Nano Lett. 2007, 7, 3134-3137.

(39) Dieguez, O.; Alemany, M.; Rey, C.; Ordejon, P.; Gallego, L. Phys. Rev. B: Condens. Matter Mater. Phys. 2001, 63, 205407.

(40) Mohn, P. Magnetism in the Solid State: An Introduction; SpringerVerlag: Berlin, 2003.

(41) Giannozzi, P.; et al. J. Phys.: Condens. Matter 2009, 21, 395502.

(42) Parsina, I.; Di Paola, C.; Baletto, F. Nanoscale 2012, 4, 1160.

(43) Di Paola, C.; Baletto, F. Proceedings for CMMSE XI 2011, 2, 451.

(44) Tang, W.; Sanville, E.; Henkelman, G. J. Phys.: Condens. Matter 2009, 21, 084204.

(45) Bader, R. Atoms in Molecules: A Quantum Theory; Oxford University Press: New York, 1990. 